\title{
The Effect of Unplanned Pregnancy on Postpartum Depression: A Meta-Analysis
}

\author{
Dinda Anindita Salsabilla' ${ }^{1)}$, Hanung Prasetya ${ }^{\text {(), }}$, Bhisma Murti1) \\ 1)Masters Program in Public Health, Universitas Sebelas Maret \\ ${ }^{2)}$ Study Program of Acupunture, School of Health Polytechnics, Ministry of Health, Surakarta
}

\section{ABSTRACT}

Background: World data shows that depression has a 50\% higher percentage to accur in women due to hormonal, genetic, psychosocial and social stress changes. World data also shows that $13 \%$ of postpartum women experience depression. In developing countries, postpartum depression was high, which is at $19.8 \%$. One of the risk factors that influence the occurrence of postpartum depression is unplanned pregnancy. This study aims to estimate the magnitude of the effect of unplanned pregnancy on postpartum depression based on a number of previous similar studies.

Subjects and Method: This was a systematic review and meta-analysis. The articles used in this study were obtained from several databases, including PubMed, Google Scholar, and Springer Link. The articles used in this research were articles published from 2005-2020. The article search was carried out by considering the eligibility criteria defined using PICO. The population in this study included postpartum mothers with intervention, namely unplanned pregnancy, the comparison namely planned pregnancy and the outcome was postpartum depression. The keywords to look for articles were: "unplanned Pregnancy" AND "postpartum depression OR“" postnatal depression” "unplanned pregnancy" AND "postpartum depression" AND "adjusted odds ratio". The articles included in this study were full text articles with an observational study design. Articles were collected using PRISMA flow diagrams. Articles were analyzed using the Review Manager 5.3 application.

Results: There are 17 articles reviewed in this study. Meta-analysis of 4 cohort studies show that unplanned pregnancy increased the risk of postpartum depression $(\mathrm{aOR}=1.27 ; 95 \% \mathrm{CI}=$ 1.09 to $1.47 ; \mathrm{p}$ <0.001). A meta-analysis of 13 cross-sectional studies show that unplanned pregnancy increased the risk of postpartum depression $(\mathrm{aOR}=2.28 ; 95 \% \mathrm{CI}=1.67$ to $3.12 ; \mathrm{p}$ $<0.001)$.

Conclusion: An unplanned pregnancy increases the risk of postpartum depression.

Keywords: Unplanned pregnancy, postpartum depression, postnatal depression

\section{Correspondence:}

Dinda Anindita Salsabilla. Masters Program in Public Health, Universitas Sebelas Maret, Jl. Ir. Sutami 36A, Surakarta 57126, Ccentral Java. Email: dindaaninditasalsa@gmail.com.

\section{Cite this as:}

Salsabilla DA, Prasetya H, Murti B (2020). The Effect of Unplanned Pregnancy on Postpartum Depression: A Meta-Analysis. J Matern Child Health. 05(05): 500-513. https://doi.org/10.26911/thejmch.2020.05.05.05.

cc (†) (2) Journal of Maternal and Child Health is licensed under a Creative Commons Attribution-NonCommercial-ShareAlike 4.0 International License.

\section{BACKGROUND}

Depression is a common mental disorder that usually appear with several symptoms including a depressed mood, loss of interest or pleasure, decreased energy, feelings of guilt or low self-esteem, sleep or appetite disorder, decreased concentration and also often accompanied by anxiety. Depression can become chronic and can interfere with an individual's ability to take care of his/her daily activities/responsibilities. Depression can lead to suicide. Nearly 1 million people are lost each year by suicide. 
Depression is a contributor to disease burden, economic burden as well as a cause of disability for the whole world. World data shows that depression has a 50\% higher percentage to occur in women (WFMH, 2012).

The latest research on depression in Indonesia was conducted by Peltzerdan Pengpid (2018) who found that 21.8\% of study subjects aged $>15$ years experienced moderate and severe depression. Of this prevalence, women have a higher percentage of $22.3 \%$.

Albert (2015) states that depression in women is related to hormonal changes that occur during puberty, before menstruation, during pregnancy, after childbirth and premenopause. Other factors that cause depression are genetic, psychosocial and social stressors. Mughal et al. (2020) states that the incidence of depression is quite high, one of which is in the postpartum period.

World data show that $13 \%$ of postpartum women experience depression. In developing countries, the condition is higher at $19.8 \%$ (WHO, 2018). This condition results from a series of changes both at the biological level (changes in physical fitness and hormonal changes) and at the psychological level (role as new parents and changes in marital and sexual life) so that psychopathological manifestations are more likely than this period (Ferreira et al. 2016).

Globally, depression is one of the leading causes of disease burden for women aged 14-44 years. If it occurs in the mother (maternal), this can have a negative impact on the growth, development, and child care processes with serious health implications in terms of physical, cognitive and emotional well-being during important stages such as the first 1000 days and early childhood. Depressed mothers will be disturbed in practices related to breastfeeding, health care, baby safety and development (Madlala and Kassier, 2018).

Postpartum depression is influenced by various factors. One of them is an unplanned pregnancy. Several studies have been conducted to determine the effect of unplanned pregnancy on postpartum depression, including by Koutra et al. (2016), which states that postpartum mothers whose pregnancies are not planned have a 1.25 risk of experiencing postpartum depression compared to mothers whose pregnancies are planned. Qandil et al. (2016), also stated that postpartum mothers whose pregnancies were not planned were 2.34 times more likely to experience postpartum depression than mothers whose pregnancies were planned. Filha et al. (2016), stated that postpartum mothers whose pregnancies were not planned were 1.23 times more likely to experience postpartum depression than mothers whose pregnancies were planned.

The number of primary studies examining the effect of unplanned pregnancy on postpartum depression has prompted researchers to conduct a more comprehensive study of these primary studies. The data obtained were analyzed using a systematic review and meta-analysis by synthesizing the results of studies conducted to reduce bias.

\section{SUBJECTS AND METHOD}

\section{Study Design}

This study uses a systematic review and meta-analysis. The articles used in this study were obtained from several databases including PubMed, Google Scholar, and Springer Link. The keywords used to find the article were as follows: "unplanned Pregnancy" AND "postpartum depression OR“ postnatal depression "“ unplanned 
pregnancy "AND“ postpartum depression "AND“ adjusted odds ratio".

\section{Inclusion Criteria}

The articles included in this study are full paper articles with an observational study design and in English. Appropriate articles should mention unplanned pregnancy interventions with the outcome of postpartum depression. Articles should be properly titled with risk factors for postpartum depression. The article used a standardized depression scale with the appropriate population, namely postpartum mothers. The analysis used is the multivariate aOR.

\section{Exclusion Criteria}

The articles published in this study are articles that have been carried out by metaanalysis. Articles published in other languages and not in English. Articles showed results which were not adjusted Odds Ratio.

\section{Operational Definition}

The articles were carried out by considering the eligibility criteria defined using PICO model. The population in this study were postpartum mothers with intervention in the form of unplanned pregnancy, comparison of planned pregnancies and postpartum depression outcomes.

Unplanned pregnancy was a pregnancy that can occur in all age groups of women but at unexpected or inaccurate times.

Postpartum depression was a mood disorder characterized by feelings of laziness, loss of interest, enthusiasm, guilt or low self-esteem, sleep disorders, appetite disorders, and poor concentration that occur during the puerperium.

\section{Data Analysis}

Data processing was performed using the Review Manager (RevMan 5.3) by calcu- lating the effect size and heterogeneity to determine which research models were combined to form the final meta-analysis result.

\section{RESULTS}

The process of searching for articles is by searching through a database with journals which can be seen in Figure 1. Figure 2 shows the areas where articles were taken according to the inclusion criteria. Articles were obtained from 6 continents, namely Asia, Africa, Europe, North America, South America and Australia.

Based on the results of the forest plot (Figure 3) in the cohort study, postpartum mothers with unplanned pregnancies had a 1.27 times risk of experiencing postpartum depression compared to postpartum mothers with planned pregnancies and this was statistically significant ( $p<0.001$ ). The heterogeneity of the research data shows that $\mathrm{I}^{2}=0 \%$. Whereas in a cross-sectional study, postpartum mothers with unplanned pregnancies had 2.28 times the risk of experiencing postpartum depression compared to postpartum mothers with planned pregnancies and it was statistically significant ( $p<0.001$ ). The heterogeneity of the research data showed $\mathrm{I}^{2}=86 \%$. So that the distribution of data is declared heterogeneous (random effect model).

The funnel plot (Figure 4) does not show any publication bias, indicated by the symmetrical plot on the right and left, where 9 plots are on the left and 8 are on the right. The plot on the left of the graph appears to have a standard error between 0 and 1 and the plot on the right has a standard error between 0 and 0.5 . 
Salsabilla et al./ Unplanned Pregnancy and Postpartum Depression

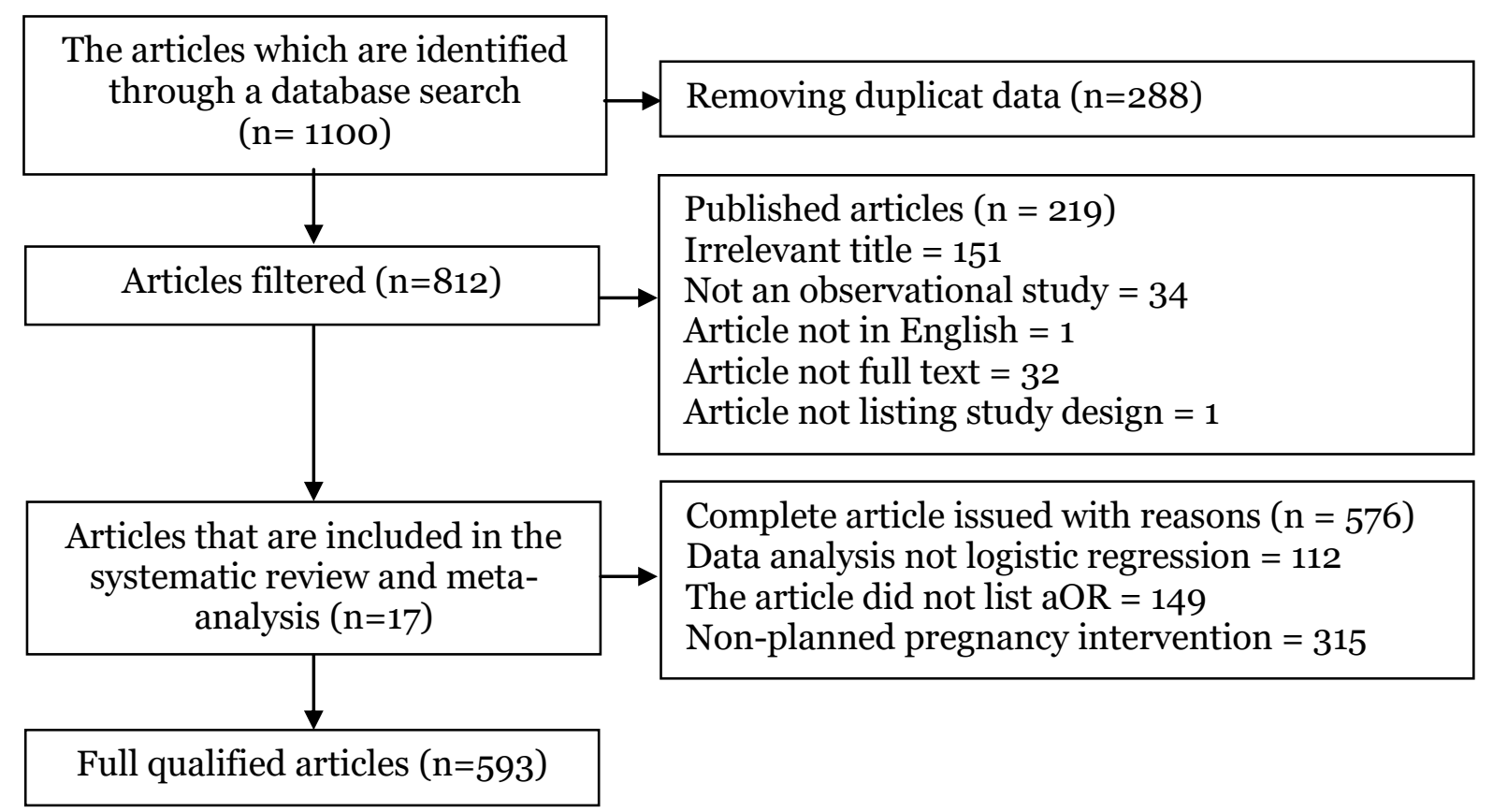

Figure 1. PRISMA flow diagram

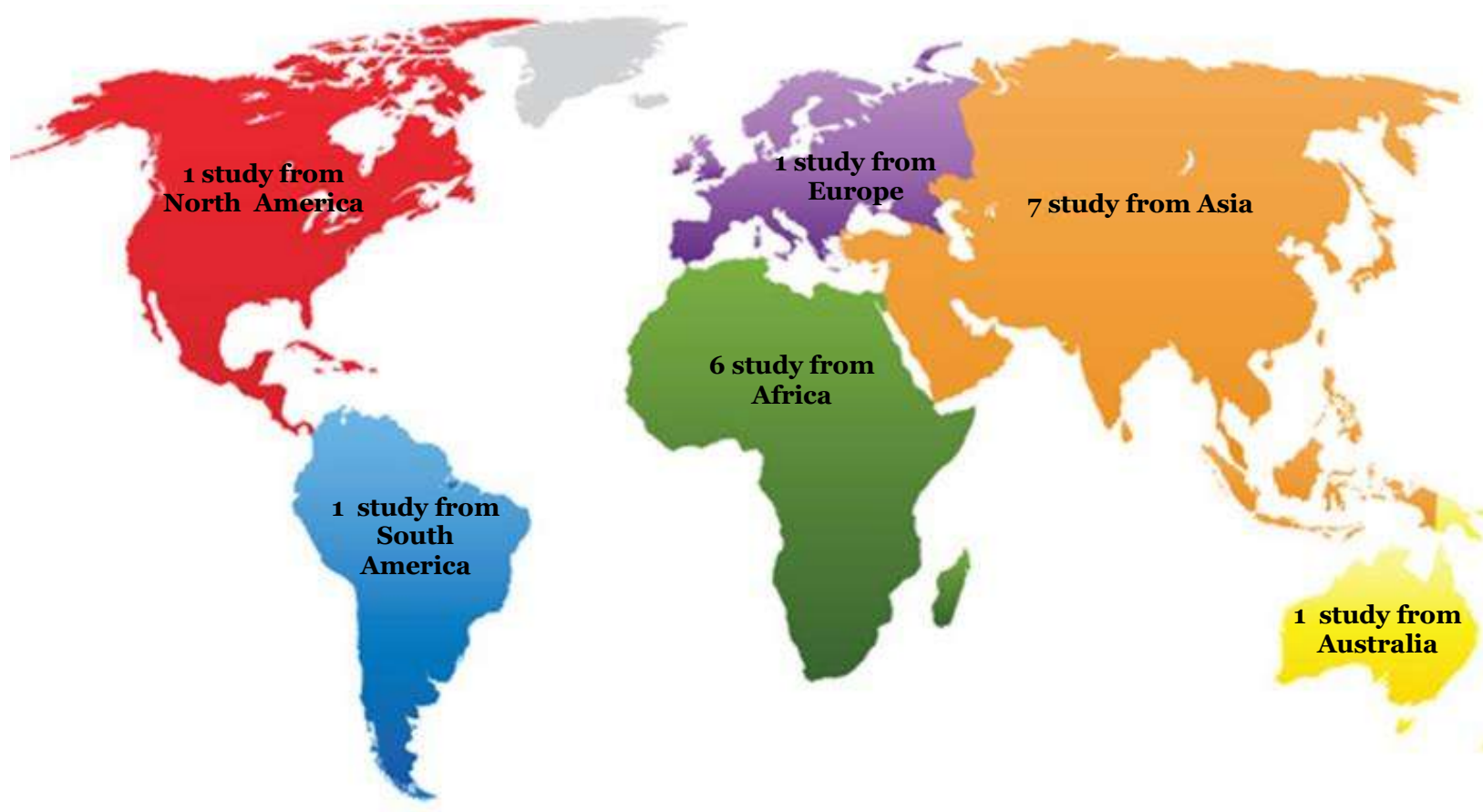

Figure 2. Research Areas Map 
Salsabilla et al./ Unplanned Pregnancy and Postpartum Depression

1. Unplanned pregnancy against postpartum depression

\section{a. Forest Plot}

Odds Ratio

Odds Ratio

Study or Subgroup log[Odds Ratio] SE Weight IV, Random, 95\% Cl IV, Random, $95 \% \mathrm{Cl}$

\begin{tabular}{|c|c|c|c|c|}
\hline Cankaya 2020 & 0.2624 & 1.0379 & $1.1 \%$ & $1.30[0.17,9.94]$ \\
\hline Filha 2016 & 0.2151 & 0.0849 & $8.9 \%$ & $1.24[1.05,1.46]$ \\
\hline Koutra 2016 & 0.2231 & 0.2028 & $7.3 \%$ & $1.25[0.84,1.86]$ \\
\hline $\begin{array}{l}\text { Qandil } 2016 \\
\text { Subtotal }(95 \% \mathrm{Cl})\end{array}$ & 0.892 & 0.4602 & $\begin{array}{r}3.8 \% \\
21.1 \%\end{array}$ & $\begin{array}{l}2.44[0.99,6.01] \\
1.27[1.09,1.47]\end{array}$ \\
\hline \multicolumn{5}{|c|}{$\begin{array}{l}\text { Heterogeneity: } \mathrm{Tau}^{2}=0.00 ; \mathrm{Chi}^{2}=2.10, \mathrm{df}=3(\mathrm{P}=0.55) ; \mathrm{I}^{2}=0 \% \\
\text { Test for overall effect: } \mathrm{Z}=3.06(\mathrm{P}=0.002)\end{array}$} \\
\hline \multicolumn{5}{|c|}{ 1.1.2 Cross-sectional } \\
\hline Abadiga 2019 & 2.0592 & 0.4588 & $3.8 \%$ & $7.84[3.19,19.27]$ \\
\hline Abebe 2019 & 0.6206 & 0.3065 & $5.7 \%$ & $1.86[1.02,3.39]$ \\
\hline Ahmad 2018 & 1.2 & 0.1763 & $7.7 \%$ & $3.32[2.35,4.69]$ \\
\hline Arifin 2014 & 0.7129 & 0.2581 & $6.4 \%$ & $2.04[1.23,3.38]$ \\
\hline Asaye 2020 & 0.7031 & 0.249 & $6.5 \%$ & $2.02[1.24,3.29]$ \\
\hline Burgut 2013 & 0.5423 & 0.247 & $6.6 \%$ & $1.72[1.06,2.79]$ \\
\hline Castro 2014 & 0.8755 & 0.3537 & $5.0 \%$ & $2.40[1.20,4.80]$ \\
\hline Dlamini 2019 & 0.6729 & 0.511 & $3.3 \%$ & $1.96[0.72,5.34]$ \\
\hline Eastwood 2011 & 0.1823 & 0.0444 & $9.2 \%$ & $1.20[1.10,1.31]$ \\
\hline Kadir 2009 & 0.1823 & 0.2536 & $6.5 \%$ & $1.20[0.73,1.97]$ \\
\hline Kerie 2018 & 1.5019 & 0.3391 & $5.2 \%$ & $4.49[2.31,8.73]$ \\
\hline Toru 2018 & 1.209 & 0.3461 & $5.1 \%$ & $3.35[1.70,6.60]$ \\
\hline $\begin{array}{l}\text { Xiong } 2018 \\
\text { Subtotal }(95 \% \mathrm{Cl})\end{array}$ & 0.8544 & 0.1592 & $\begin{array}{r}8.0 \% \\
78.9 \%\end{array}$ & $\begin{array}{l}2.35[1.72,3.21] \\
2.28[1.67,3.12]\end{array}$ \\
\hline \multicolumn{5}{|c|}{$\begin{array}{l}\text { Heterogeneity: } \text { Tau }^{2}=0.25 ; \mathrm{Chi}^{2}=87.46, \mathrm{df}=12(\mathrm{P}<0.00001) ; \mathrm{I}^{2}=86 \% \\
\text { Test for overall effect: } Z=5.18(\mathrm{P}<0.00001)\end{array}$} \\
\hline Total $(95 \% \mathrm{Cl})$ & & & $100.0 \%$ & $2.03[1.62,2.55]$ \\
\hline \multicolumn{5}{|c|}{ Heterogeneity: $\mathrm{Tau}^{2}=0.14 ; \mathrm{Chi}^{2}=91.69, \mathrm{df}=16(\mathrm{P}<0.00001) ; \mathrm{I}^{2}=83 \%$} \\
\hline
\end{tabular}

Figure 3. Forest plot of the effect of unplanned pregnancy on postpartum depression

\section{b. Funnel Plot}

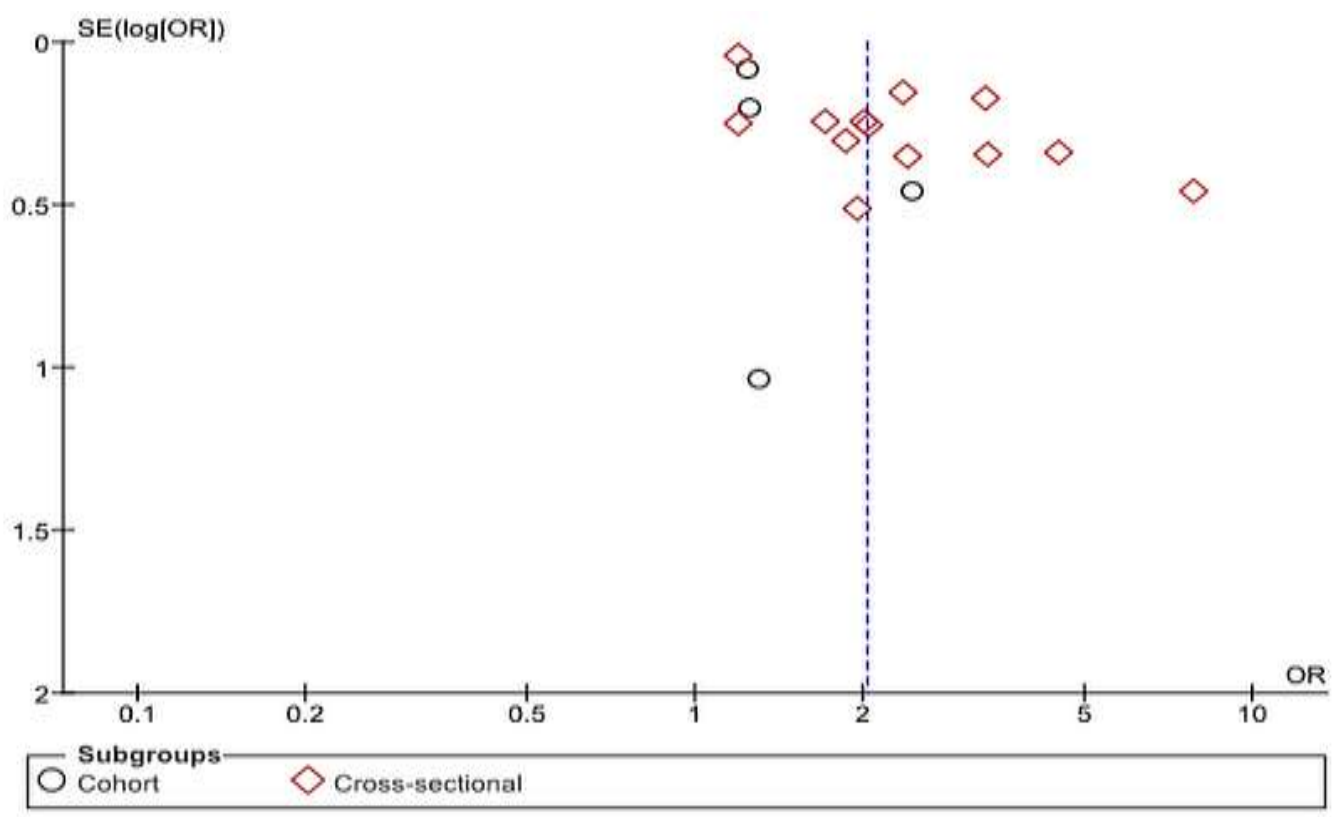

Figure 4. Funnel plot of the effect of unplanned pregnancy against postpartum depression 


\section{Checklists Questions}

Does the research address clearly focused issues?

Was the group included in an acceptable way?

Is exposure measured accurately to minimize bias?

Are the results measured accurately to minimize bias?

Have the authors identified all the important confounding factors?

Was the subject follow-up of the study complete?

What are the results of this study?

How precise is the result?

Do you believe in the results?

Can the results be applied to the local population?

Are the results of this study consistent with other available evidence?

What are the implications of this study for practice?

\section{Table 2. Critical appraisal checklist for cross-sectional study}

\section{Checklists Questions

Does this research address
clearly focused issues?
Are the groups entered in an
acceptable manner?
Are the exposures measured
accurately to minimize bias?
Are the results accurately
assessed to minimize bias?
Has the author identified all
the important confounding
factors?
Was the follow-up subject of
this research complete?

Publication

\begin{tabular}{cccc}
\multicolumn{4}{c}{ Author and Year } \\
\hline $\begin{array}{c}\text { Qandil et al. } \\
\text { (2016) }\end{array}$ & $\begin{array}{c}\text { Filha et al. } \\
\text { (2016) }\end{array}$ & $\begin{array}{c}\text { Cankaya et } \\
\text { al. (2020) }\end{array}$ & $\begin{array}{c}\text { Koutra et } \\
\text { al. (2020) }\end{array}$ \\
\hline 1 & 1 & 1 & 1 \\
1 & 1 & 1 & 1 \\
1 & 1 & 1 & 1 \\
1 & 1 & 1 & 1 \\
1 & 1 & 1 & 1 \\
0 & 1 & 0 & 0 \\
1 & 1 & 1 & 1 \\
1 & 1 & 1 & 1 \\
1 & 1 & 1 & 1 \\
0 & 1 & 1 & 1 \\
1 & 1 & 1 & 1 \\
1 & 1 & 1 & 11 \\
10 & 11 & 11 & \\
\hline
\end{tabular}

Qandil et Filha et al. Cankaya et Koutra et

\section{Author and Year}

al. (2016) $\quad$ (2016) $\quad$ al. (2020) $\quad$ al. (2020)

$\begin{array}{ccc}(2016) & \text { (2016) } & \text { al. ( } \\ 1 & 1 \\ 1 & 1 \\ 1 & 1 \\ 1 & 1 \\ 1 & 1\end{array}$

O

1

1

al. (2014)
Abebe et al. Asaye et (2019) al. (2020)

1

1

1

1

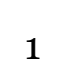

1

O

O

1

1

O

o 
Salsabilla et al./ Unplanned Pregnancy and Postpartum Depression

What are the results of this

research?

How successful has it been?

Do you believe in the results?

Can the results be applied to

local populations?

Do the results of this study

match other available

evidence?

What are the implications of

this study for practice?

Total

Note: Yes $=1, \mathrm{No}=0$

$1 \quad 1$

1

11

1
1
1

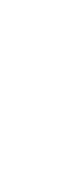

1

1

1

1

1

1

11

11

ta

Table 3.Critical appraisal checklist for cross-sectional study Checklists Questions

\begin{tabular}{|c|c|c|c|c|c|c|}
\hline \multirow[t]{2}{*}{ Checklists Questions } & \multicolumn{6}{|c|}{$\begin{array}{c}\text { Publication } \\
\text { (Author and Year) }\end{array}$} \\
\hline & $\begin{array}{l}\text { Dlamini et } \\
\text { al. (2019) }\end{array}$ & $\begin{array}{l}\text { Arifin et } \\
\text { al. (2014) }\end{array}$ & $\begin{array}{l}\text { Xiong et } \\
\text { al. (2018) }\end{array}$ & $\begin{array}{c}\text { Ahmad et al. } \\
\text { (2018) }\end{array}$ & $\begin{array}{l}\text { Kadir et al. } \\
\text { (2009) }\end{array}$ & $\begin{array}{l}\text { Eastwood et } \\
\text { al. (2011) }\end{array}$ \\
\hline $\begin{array}{l}\text { Does this objective clearly address the } \\
\text { focus / research problem? }\end{array}$ & 1 & 1 & 1 & 1 & 1 & 1 \\
\hline $\begin{array}{l}\text { Is the cross-sectional research method } \\
\text { suitable for answering research } \\
\text { questions? }\end{array}$ & 1 & 1 & 1 & 1 & 1 & 1 \\
\hline $\begin{array}{l}\text { Is the method of selecting research } \\
\text { subjects clearly written? }\end{array}$ & 1 & 1 & 1 & 1 & 1 & 1 \\
\hline $\begin{array}{l}\text { Does the sampling method create bias } \\
\text { (selection)? }\end{array}$ & 1 & 1 & 1 & 1 & 1 & 1 \\
\hline $\begin{array}{l}\text { Does the research sample taken } \\
\text { represent the designated population? }\end{array}$ & 1 & 1 & 1 & 1 & 1 & 1 \\
\hline $\begin{array}{l}\text { Was the sample size based on pre-study } \\
\text { considerations? }\end{array}$ & 0 & 0 & 0 & $\mathrm{o}$ & 0 & 0 \\
\hline Was a satisfactory response achieved? & 1 & 1 & 1 & 1 & 1 & 1 \\
\hline $\begin{array}{l}\text { Are the research instruments valid and } \\
\text { reliable? }\end{array}$ & 1 & 1 & 1 & 1 & 1 & 1 \\
\hline Is statistical significance assessed? & 1 & 1 & 1 & 1 & 1 & 1 \\
\hline Are confidence intervals given for the & 1 & 1 & 1 & 1 & 1 & 1 \\
\hline
\end{tabular}

www.thejmch.com 
Salsabilla et al./ Unplanned Pregnancy and Postpartum Depression

main outcome?

Are there any confounding factors that

o

o

O

O

have not been taken into account?

Are the results applicable to your

1

1

1

1

research?

10

10

10

10

10

10

Note: $\mathrm{Yes}=1, \mathrm{No}=\mathrm{O}$

Table 4. Primary study descriptions were included in the meta-analysis

\begin{tabular}{ccccccc}
$\begin{array}{c}\text { Author } \\
\text { (year) }\end{array}$ & Country & $\begin{array}{c}\text { Study } \\
\text { Design }\end{array}$ & Sample & $\begin{array}{c}\text { P } \\
\text { Population }\end{array}$ & $\begin{array}{c}\text { I } \\
\text { Intervention }\end{array}$ & $\begin{array}{c}\text { C } \\
\text { Comparison }\end{array}$ \\
\hline
\end{tabular}

al. (2016) mothers nancy program, hypertension / pre-
eclampsia, smoking during pregnancy, alcohol consumption during pregnancy, weight gain, lack of sleep, caesasoon, no hypertension/pre-eclampsia, depression, no smoking, no alcohol consumption, EPDS $\geq 13$ no weight gain, no sleep deprivation, normal delivery, can breastfeed

\begin{tabular}{|c|c|c|}
\hline Qandil et & Palestina & Kohor \\
\hline
\end{tabular}

rean delivery, difficulty breastfeeding

Place of delivery in hospital, cesarean delivery, unplanned pregnancy*, gender of girl, gender of child is

Place of birth at home, normal childbirth, planned pregnancy, gender of

Postpartum unwanted,

Filha et Brazil Kohor 23,894 Postpartum

Brown skin color, low economic sta-

al. (2016)

mothers

$\begin{array}{lllll}\begin{array}{l}\text { Çankaya } \\ (2020)\end{array} & \text { Turki } & \text { Kohor } & 245 & \begin{array}{l}\text { Postpartum } \\ \text { mothers }\end{array}\end{array}$

tus, history of chronic illness, alcoho consumption, parity $\geq 3$, unplanned pregnancy*, poor newborn care

ANC was not routine, pregnancy was not planned*, husband did not want pregnancy, experienced violence, there was a history of family psychological problems, smoking during pregnancy, there was a history of abortion, hyperemesis, dissatisfaction with the gender of the baby

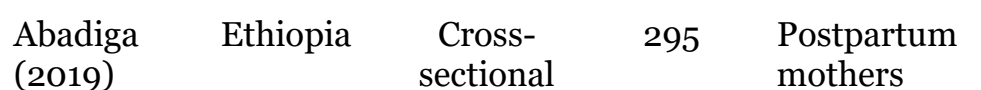

Maternal age 15-24 years, single status, daily labor, income $<500 \mathrm{~EB}$, unplanned pregnancy*, premature or fetal death, parity 1, cesarean delivery, history of abortion, chronic illness, boy, gender of child desired

White skin color, high economic status, no history of chronic disease, no alcohol consumption, 1-2 parity, good newborn care

Routine ANC, planned pregnancy, husband wants a pregnancy, does not experience violence, no family history of psychological problems, does not smoke during pregnancy, no history of abortion, no hyperemesis, satisfied with the gender of the baby

Maternal age> 25 years, married status, government employee occupation, income $>500 \mathrm{~EB}$, planned pregnancy, not premature, parity $>2$, normal delivery, no history of abort-

EPDS $\geq 11$

Postpartum depression, EPDS $\geq 13$

Postpartum depression, EPDS $\geq 13$

Postpartum depression,

EPDS $\geq 10$ 
Salsabilla et al./ Unplanned Pregnancy and Postpartum Depression

\begin{tabular}{|c|c|c|c|c|c|c|c|}
\hline $\begin{array}{l}\text { Author } \\
\text { (year) }\end{array}$ & Country & $\begin{array}{c}\text { Study } \\
\text { Design }\end{array}$ & Sample & $\begin{array}{c}\mathbf{P} \\
\text { Population }\end{array}$ & $\begin{array}{c}\text { I } \\
\text { Intervention }\end{array}$ & $\begin{array}{c}\mathrm{C} \\
\text { Comparison }\end{array}$ & $\begin{array}{c}\text { O } \\
\text { Outcome } \\
\text { and scale }\end{array}$ \\
\hline & & & & & $\begin{array}{l}\text { history of depression, domestic vio- } \\
\text { lence, low social support. }\end{array}$ & $\begin{array}{l}\text { ion, no chronic disease, no history of } \\
\text { depression, no internal violence } \\
\text { household, good social support }\end{array}$ & \\
\hline $\begin{array}{l}\text { Kerie et } \\
\text { al. } \\
\text { (2018) }\end{array}$ & Ethiopia & $\begin{array}{c}\text { Cross- } \\
\text { sectional }\end{array}$ & 422 & $\begin{array}{l}\text { Postpartum } \\
\text { mothers }\end{array}$ & $\begin{array}{l}\text { Maternal age }>30 \text { years, unplanned } \\
\text { pregnancy*, chronic illness, history of } \\
\text { depression, fetal death, marital } \\
\text { problems }\end{array}$ & $\begin{array}{l}\text { Maternal age } 15-24 \text { years, planned } \\
\text { pregnancy, no chronic illness, no } \\
\text { history of depression, no fetal death, } \\
\text { no marital problems }\end{array}$ & $\begin{array}{l}\text { Postpartum } \\
\text { depression, } \\
\text { EPDS } \geq 10\end{array}$ \\
\hline $\begin{array}{l}\text { Toru T et } \\
\text { al. (2018) }\end{array}$ & Ethiopia & $\begin{array}{c}\text { Cross- } \\
\text { sectional }\end{array}$ & 460 & $\begin{array}{l}\text { Postpartum } \\
\text { mothers }\end{array}$ & $\begin{array}{l}\text { Age } 18-23 \text { years, unplanned preg- } \\
\text { nancy*, domestic violence, marital } \\
\text { dissatisfaction, poor social support, } \\
\text { history of depression }\end{array}$ & $\begin{array}{l}\text { Age }>24 \text { years, planned pregnancy, no } \\
\text { domestic violence, satisfied in } \\
\text { marriage, good social support, no } \\
\text { history of depression }\end{array}$ & $\begin{array}{l}\text { Postpartum } \\
\text { depression, } \\
\text { PHQ-9 } \geq 10\end{array}$ \\
\hline $\begin{array}{l}\text { Burgut et } \\
\text { al. (2013) }\end{array}$ & Qatar & $\begin{array}{c}\text { Cross- } \\
\text { sectional }\end{array}$ & 1,379 & $\begin{array}{l}\text { Postpartum } \\
\text { mothers }\end{array}$ & $\begin{array}{l}\text { Age }<25 \text { years, education level } \\
<\text { secondary, housewife work, income } \\
<10, \text { ooo QR, unplanned pregnancy*, } \\
\text { caesarean delivery, using formula milk }\end{array}$ & $\begin{array}{l}\text { Age }>25 \text { years, education level } \\
\geq \text { secondary, work outside the home, } \\
\text { income } \geq 10 \text {,ooo } Q R \text {, planned preg- } \\
\text { nancy, normal delivery, breastfeeding. }\end{array}$ & $\begin{array}{l}\text { Postpartum } \\
\text { depression, } \\
\text { EPDS } \geq 12\end{array}$ \\
\hline $\begin{array}{l}\text { Castro et } \\
\text { al. (2014) }\end{array}$ & Meksiko & $\begin{array}{l}\text { Cross- } \\
\text { sectional }\end{array}$ & 604 & $\begin{array}{l}\text { Postpartum } \\
\text { mothers }\end{array}$ & $\begin{array}{l}\text { Maternal age }<20 \text { years, single status, } \\
\leq \text { basic level of education, low social } \\
\text { support, history of depression, } \\
\text { unplanned pregnancy*, experiencing } \\
\text { domestic violence }\end{array}$ & $\begin{array}{l}\text { Maternal age } \geq 20 \text { years, married } \\
\text { status, education level >basic, high } \\
\text { social support, no history of depress- } \\
\text { ion, planned pregnancy, no domestic } \\
\text { violence }\end{array}$ & $\begin{array}{l}\text { Postnatal } \\
\text { depression, } \\
\text { EPDS } \geq 12\end{array}$ \\
\hline $\begin{array}{l}\text { Abebe et } \\
\text { al. (2019) }\end{array}$ & Ethiopia & $\begin{array}{c}\text { Cross- } \\
\text { sectional }\end{array}$ & 511 & $\begin{array}{l}\text { Postpartum } \\
\text { mothers }\end{array}$ & $\begin{array}{l}\text { No decision making rights, unplanned } \\
\text { pregnancy*, domestic violence, history } \\
\text { of depression }\end{array}$ & $\begin{array}{l}\text { Decision-making rights, planned } \\
\text { pregnancy, no domestic violence, no } \\
\text { history of depression }\end{array}$ & $\begin{array}{l}\text { Postpartum } \\
\text { depression, } \\
\text { EPDS } \geq 13\end{array}$ \\
\hline $\begin{array}{l}\text { Asaye et } \\
\text { al. } \\
\text { (2020) }\end{array}$ & Ethiopia & $\begin{array}{c}\text { Cross- } \\
\text { sectional }\end{array}$ & 526 & $\begin{array}{l}\text { Postpartum } \\
\text { mothers }\end{array}$ & $\begin{array}{l}\text { Child alive } \geq 2 \text {, history of abortion, } \\
\text { unplanned pregnancy*, pregnancy } \\
\text { complications, ANC visits, PNC visits }\end{array}$ & $\begin{array}{l}\text { Child alive }<2 \text {, no history of abortion, } \\
\text { planned pregnancy, no pregnancy } \\
\text { complications, no ANC visit, no PNC } \\
\text { visit }\end{array}$ & $\begin{array}{l}\text { Postpartum } \\
\text { depression, } \\
\text { EPDS } \geq 13\end{array}$ \\
\hline $\begin{array}{l}\text { Xiong et } \\
\text { al. (2018) }\end{array}$ & Cina & $\begin{array}{c}\text { Cross- } \\
\text { sectional }\end{array}$ & 468 & $\begin{array}{l}\text { Postpartum } \\
\text { mothers }\end{array}$ & $\begin{array}{l}\text { Unplanned pregnancy*, poor relation- } \\
\text { ship with in-laws, lack of family } \\
\text { support, parity } 1\end{array}$ & $\begin{array}{l}\text { Pregnancy planned, good relationship } \\
\text { with in-laws, always receive family } \\
\text { support, parity }>1\end{array}$ & $\begin{array}{l}\text { Postpartum } \\
\text { depression, } \\
\text { EPDS } \geq 10\end{array}$ \\
\hline $\begin{array}{l}\text { Ahmad et } \\
\text { al. (2018) }\end{array}$ & Malaysia & $\begin{array}{c}\text { Cross- } \\
\text { sectional }\end{array}$ & 5,727 & $\begin{array}{l}\text { Postpartum } \\
\text { mothers }\end{array}$ & $\begin{array}{l}\text { Experiencing domestic violence, expe- } \\
\text { riencing emotional violence, unplan- } \\
\text { ned pregnancy*, lack of family sup- } \\
\text { port, income }<1500 \mathrm{RM}\end{array}$ & $\begin{array}{l}\text { Not experiencing domestic violence, } \\
\text { not experiencing emotional violence, } \\
\text { planned pregnancy, good family } \\
\text { support, income } \geq 15 \text { oo RM }\end{array}$ & $\begin{array}{l}\text { Postnatal } \\
\text { depression, } \\
\text { EPDS } \geq 12\end{array}$ \\
\hline $\begin{array}{l}\text { Dlamini } \\
\text { et al. } \\
\text { (2019) }\end{array}$ & Eswatini & $\begin{array}{c}\text { Cross- } \\
\text { sectional }\end{array}$ & 114 & $\begin{array}{l}\text { Postpartum } \\
\text { mothers }\end{array}$ & $\begin{array}{l}\text { Not working, unplanned pregnancy*, } \\
\text { poor husband support, history of } \\
\text { gender based violence, presence of } \\
\text { ANC }<4 \text { times }\end{array}$ & $\begin{array}{l}\text { Work, planned pregnancy, husband } \\
\text { support, no history of gender-based } \\
\text { violence, presence of ANC } \geq 4 \text { times }\end{array}$ & $\begin{array}{l}\text { Postpartum } \\
\text { depression, } \\
\text { EPDS } \geq 13\end{array}$ \\
\hline \multicolumn{3}{|c|}{ www.thejmch.com } & \multicolumn{2}{|c|}{ www.thejmch.com } & & & 508 \\
\hline
\end{tabular}


Salsabilla et al./ Unplanned Pregnancy and Postpartum Depression

\begin{tabular}{|c|c|c|c|c|c|c|c|}
\hline $\begin{array}{l}\text { Author } \\
\text { (year) }\end{array}$ & Country & $\begin{array}{l}\text { Study } \\
\text { Design }\end{array}$ & Sample & $\begin{array}{c}\mathbf{P} \\
\text { Population }\end{array}$ & $\begin{array}{c}\text { I } \\
\text { Intervention }\end{array}$ & $\begin{array}{c}\mathrm{C} \\
\text { Comparison }\end{array}$ & $\begin{array}{c}\text { O } \\
\text { Outcome } \\
\text { and scale }\end{array}$ \\
\hline $\begin{array}{l}\text { Arifin et } \\
\text { al.(2014) }\end{array}$ & Malaysia & $\begin{array}{c}\text { Cross- } \\
\text { sectional }\end{array}$ & 347 & $\begin{array}{l}\text { Postpartum } \\
\text { mothers }\end{array}$ & $\begin{array}{l}\text { Housewives, experience of giving } \\
\text { birth, LAS score } \leq 100 \text {, unplanned } \\
\text { pregnancy* }\end{array}$ & $\begin{array}{l}\text { Work, delivery experience, LAS score } \\
>100 \text {, planned pregnancy }\end{array}$ & $\begin{array}{l}\text { Postpartum } \\
\text { depression, } \\
\text { EPDS } \geq 11\end{array}$ \\
\hline $\begin{array}{l}\text { Kadir et } \\
\text { al. } \\
(2009)\end{array}$ & Malaysia & $\begin{array}{c}\text { Cross } \\
\text { sectional }\end{array}$ & 293 & $\begin{array}{l}\text { Postpartum } \\
\text { mothers }\end{array}$ & $\begin{array}{l}\text { Parity }>5 \text {, there is a history of abort- } \\
\text { ion, childbirth with tools, pregnancy } \\
\text { complications, postnatal complica- } \\
\text { tions, unplanned pregnancy * }\end{array}$ & $\begin{array}{l}\text { Parity }<5 \text {, no history of abortion, cesa- } \\
\text { rean delivery, no pregnancy complica- } \\
\text { tions, no postnatal complications, } \\
\text { planned pregnancy }\end{array}$ & $\begin{array}{l}\text { Postnatal } \\
\text { depression, } \\
\text { EPDS } \geq 12\end{array}$ \\
\hline $\begin{array}{l}\text { Eastwood } \\
\text { et al. } \\
\text { (2011) }\end{array}$ & Australia & $\begin{array}{c}\text { cross- } \\
\text { sectional }\end{array}$ & 25,455 & $\begin{array}{l}\text { Postpartum } \\
\text { mothers }\end{array}$ & $\begin{array}{l}\text { Childbirth in a country other than } \\
\text { Australia, financial hardship, unedu- } \\
\text { cated mother, single status, not } \\
\text { breastfeeding, poor self-care, un- } \\
\text { planned pregnancy*, poor child care }\end{array}$ & $\begin{array}{l}\text { Give birth in Australia, good financial, } \\
\text { highly educated mother, married } \\
\text { status, breastfeeding, good self-care, } \\
\text { planned pregnancy, good child care }\end{array}$ & $\begin{array}{l}\text { Postnatal } \\
\text { depression, } \\
\text { EPDS } \geq 9\end{array}$ \\
\hline
\end{tabular}

*variables were included in the meta-analysis study 


\section{DISCUSSION}

This research is a systematic study and meta-analysis that raises the theme of the effect of unplanned pregnancy on postpartum depression. This study discusses data on postpartum depression due to mental health which is an important but often neglected component of reproductive health (Humayun et al. 2013).

This systematic study study and metaanalysis used research that controlled for confounding factors which could be seen from the study inclusion requirements, namely multivariate analysis, and the statistical result reported was the adjusted odds ratio (aOR).

Estimates of the combined effect of unplanned pregnancy and postpartum depression were processed using the RevMan 5.3 application with the generic inverse variance method.

The results of the systematic study and meta-analysis are presented in the form of a forest plot and a funnel plot. Forest plots provide an overview of information from each of the studies examined in the meta-analysis, and estimates of the overall results (Murti, 2018).

The funnel plot shows visually the amount of variation (heterogeneity) (Akobeng, 2005 in Murti, 2018). The funnel plot shows the relationship between the effect size of the study and the sample size of the various studies studied, which can be measured in a number of different ways (Murti, 2018).

\section{Unplanned pregnancy against postpartum depression}

The results of the forest plot in the cohort study showed that postpartum mothers with unplanned pregnancies had 1.27 times the risk of experiencing postpartum depression compared to postpartum mothers with planned pregnancies. Meanwhile, a research article with a cross-sectional study showed that postpartum mothers with unplanned pregnancies were 2.17 times more likely to experience postpartum depression than postpartum mothers with planned pregnancies.

Kozinszky et al. (2011) in their study stated that postpartum mothers with unplanned pregnancies had 1.71 times the risk of experiencing postpartum depression compared to postpartum mothers with planned pregnancies and were statistically significant $(\mathrm{aOR}=1.71 ; 95 \% \mathrm{CI}=1.13$ to 2.32; $\mathrm{p}<0.001)$.

In line with these studies, Adama et al. (2015) in their research stated that postpartum mothers with unplanned pregnancies had a 1.08 times risk of experiencing postpartum depression $(\mathrm{aOR}=1.08$; 95\% CI $=0.27-4.35 ; \mathrm{p}=0.91)$.

Chen et al. (2018) also stated that postpartum mothers with unplanned pregnancies had 2.21 times the risk of experiencing postpartum depression compared to postpartum mothers whose pregnancies were planned $\left(\mathrm{aOR}=2.21 ; 95 \% \mathrm{CI}=1.34^{-}\right.$ 2.75; $\mathrm{p}<0.001$ ).

A study by Lara et al. (2016), also stated that postpartum mothers with unplanned pregnancies had a 2.81 times risk of experiencing postpartum depression $(\mathrm{aOR}=2.81 ; 95 \% \mathrm{CI}=1.36$ to 4.82$)$. Postpartum mothers with unplanned pregnancies who experience postpartum depression are more likely to occur in low- and lowermiddle income countries and minorities who are affected by other social disadvantages than are women who enjoy better socio-economic conditions.

This is different from the study of Hanlon and Beckman (2015), which states that postpartum mothers with unplanned pregnancies decreased the incidence of postpartum depression by $46 \%$ compared to postpartum mothers with planned pregnancies $(\mathrm{aOR}=0.54,95 \% \mathrm{CI}=0.29-0.97$; 
Salsabilla et al./ Unplanned Pregnancy and Postpartum Depression

$\mathrm{p}=$ o.038). This can happen because even though the postpartum mother has an unplanned pregnancy, she gets good support from her husband.

\section{AUTHOR CONTRIBUTION}

Dinda is the principal researcher who selects topics, explores and collects data. Hanung Prasetya and Bhisma Murti played a role in analyzing data and reviewing research documents

\section{CONFLICT OF INTEREST}

There is no conflict of interest in this study.

\section{FUNDING AND SPONSORSHIP}

This research is self-funded.

\section{ACKNOWLEDGEMENT}

We are very grateful to the database providers PubMed, Google Scholar and Springer Link

\section{REFERENCE}

Abadiga M (2019). Magnitude and associated factors of postpartum depression among women in Nekemte town, East Wollega zone, west Ethiopia, 2019: A community-based study. PLoS ONE, 14(11): e0224792. doi: https://doi.org/10.1371/journal.pone.0224792

Abebe A, Tesfaw G, Mulat H, Hibdye G, Yohannes K (2019). Postpartum depression and associated factors among mothers in Bahir Dar Town, Northwest Ethiopia. Ann Gen Psychiatry, 18:19. https://doi.org/10.1186/s12991-019-0244-4.

Adama ND, Foumane P, Olen JPK, Dohbit JS, Meka ENUM, dan Mboudou E (2015). Prevalence and risk factors of postpartum depression in Yaounde, Cameroon. Open J Obstet Gynecol, 5; 6o8-617. doi: http://dx.doi.org/10.4236/ojog.2015.511086.

Ahmad NA, Silim UA, Rosman A, Mohamed M, Chan YY, Kasim NM, Yusof M et al. (2018). Postnatal depression and intimate partner violence: a nationwide clinic based cross-sectional study in Malaysia. BMJ Open, 8:eo20649. doi:10.1136/bmjopen-2017-020649.

Albert PR (2015). Why is depression more prevalent in women?. J Psychiatry Neurosci, 40(4): 219-221. doi: 10.1503/jpn.150205.

Arifin SRM, Ahmad A, Rasnah A. Rahman, Loh HS, Chong GN (2014). Postpartum depression in Malaysian women: the association with the timing of pregnancy and sense of personal control during childbirth. International Journal of Academic Research, 6(3): 143-149. doi:10.7813/2075 4124.2014/6-3/B.21.

Asaye MM, Muche HA, Zelalem ED (2020). Prevalence and predictors of postpartum depression: Northwest Ethiopia. Psychiatry Journal, 2020. doi:https://doi.org/10.1155/2020/9565678.

Burgut FT, Bener A, Ghuloum S, Sheikh J (2013). A study of postpartum depression and maternal risk factors in Qatar. J Psychosom Obstet Gynaecol. 34(2): 90-97. doi: 10.3109/0167482X.2013.786036.

Cankaya S (2020). The effect of psychosocial risk factors on postpartum depression in antenatal period: A prospective study. Arch Psychiatr Nurs, 34(3):176-183. doi: 10.1016/j.apnu.2020.04.007.

Castro de F, Place JMS, Billings DL, Rivera L, Frongillo EA (2014). Risk profiles associated with postnatal depressive symptoms among women in a public 
Salsabilla et al./ Unplanned Pregnancy and Postpartum Depression

sector hospital in Mexico: the role of sociodemographic and psychosocial factors. Arch Womens Ment Health, 18(3): 463-71. doi: 10.1007/soo737014-0472-1.

CEBMa (2014). Critical Appraisal for crosssectional study. Amsterdam: Center for Evidence Based Management.

Chen L, Ding L, Qi M, Jiang C, Mao XM, Cai WZ (2018). Incidence of and social-demographic and obstetric factors associated with postpartum depression: differences among ethnic Han and Kazak women of Northwestern China. Peer J, 6: e4335. doi: 10.7717/peerj.4335.

Dadi AF, Miller ER, Mwanri L (2020). Antenatal depression and its association with adverse birth outcomes in low and middle income countries: A systematic review and meta-analysis. PLoS ONE, 15(1): 1-23. doi: 10.1371/journal.pone.0227323.

Dlamini LP, Mahanya S, Dlamini SD, Schongwe MC (2019). Prevalence and factors associated with postpartum depression at a primary healthcare facility in Eswatini. Afr J Psychiatry, 25(o). doi: https://doi.org/10.4102/sajpsychiatry.v25io.1404.

Ferreira M, Catarina C, Bruno B, João D, Claudia C (2016). Determinants of psycho-emotional postpartum changes: theeffects of self-esteem. EpSBS. 20-26. http://dx.doi.org/10.15405/epsbs.2016.11.3.

Filha MMT, Susan A, Silvana GN da G, Maria do CL (2016). Factors associated with postpartum depressive symptomatology in Brazil: The Birth in Brazil National Research Study, 2011/2012. J Affect Disord, 194(2016): 159-167. doi: 10.1016/j.jad.2016.01.020.
Hanlon AJM dan Beckmann MM (2015). Mode of birth and early postnatal psychological morbidity. Aust N Z J Obstet Gynaecol, 55: 578-583. doi: 10.1111/ajo.12387.

Kadir AA, Daud MNM, Yaacob MJ, Hussain, Nik HN (2009). Relationship between Obstetric Risk Factors and Postnatal Depression in Malaysian Women. Int . Medical J. Malaysia, 16(2): 101-106.

Kerie S, Menberu M, Niguse W (2018). Prevalence and associated factors of postpartum depression in Southwest, Ethiopia, 2017: a cross-sectional study. BMC Res Notes, 11: 623. doi: https://doi.org/10.1186/s13104-0183730-x.

Khanlari S, Bryanne BAM, Felix AO, John E (2019). Re-examination of perinatal mental health policy frameworks for women signalling distress on the Edinburgh Postnatal Depression Scale (EPDS) completed during their antenatal booking-in consultation: a call for population health intervention. BMC Pregnancy and Childbirth, 19(221): 1-11. doi: https://doi.org/10.1186/s12884-019-2378-4.

Koutra K, Vassilaki M, Georgiu V, Bitsios P, Kogevinas M, Chatzi L (2016). Pregnancy, perinatal and postpartum complications as determinants of postpartum depression: the Rhea motherchild cohort in Crete, Greece. Epidemiol Psychiatr Sci. 2016: 1-12. doi:10.1017/S2045796016001062.

Kozinszky Z, Dudas RB, Csarordai S, Devosa I, Toth E, Szabo D, Sikovanyecz J, et al. (2011). Soc Psychiatr Epidemiol, 46:413-423. doi: 10.1007/s00127-010-0206-2.

Lara MA, Navarrete L, Nieto L (2016). Prenatal predictors of postpartum depression and postpartum depres- 
sive symptoms in Mexican mothers: a longitudinal study. Arch Womens Ment Health,19(5): 825-834. doi: 10.1007/s00737-016-0623-7.

Madlala SS, Kassier SM (2018). Antenatal and postpartum depression: effects on infant and young child health and feeding practices.South Afr J Clin Nutr, 31(1): 1-7. doi: 10.1080/16070658.2017.1333753.

Mughal S, Yusra A, Waquar S (2020). Postpartum Depression. StatPearls Publishing LLC. https://www.ncbi.nlm.nih.gov/books/NBK519070/.

Murti B (2018). Prinsip dan Metode Riset Epidemiologi. Edisi ke 5. Surakarta: Program Studi Ilmu Kesehatan Masyarakat.

Peltzer K, Pengpid S (2018). High prevalence of depressive symptoms in a national sample of adults in Indonesia: Childhood adversity, sociodemographic factors and health risk behaviour. Asian $\mathrm{J}$ Psychiatr. 33 (2018): 52-59. doi: 10.1016/j.ajp.2018.03.017.

Qandil S, Samah J, Stefan W, Simon MC (2016). Postpartum depression in the OccupiedPalestinian Territory: a longitudinal study in Bethlehem. BMC Pregnancy Childb. (2016) 16:375, doi: 10.1186/s12884-016-1155-X.
Qandil S, Samah J, Stefan W, Simon MC (2016). Postpartum depression in the OccupiedPalestinian Territory: a longitudinal study in Bethlehem. BMC Pregnancy Childb. (2016) 16:375, doi: 10.1186/s12884-016-1155-x.

Stellenberg EM, Abrahams JM (2015). Prevalence of and factors influencing postnatal depression in a rural community in South Africa. Afr J Prm Health Care Fam Med, 7(1). doi: http://dx.doi.org/10.4102/phcfm.v7i1.874

Toru T, Chemir F, Anand S (2018). Magnitude of postpartum depression and associated factors among women in Mizan Aman town, Bench Maji zone, Southwest Ethiopia. BMC Pregnancy and Childbirth, 18:442. doi: https://doi.org/10.1186/s12884-018-2072-y.

WFMH (2012). Depression: A Global Crisis. USA. World Federation for Mental Health.

Xiong R, Deng A, Wan B, Liu W (2018). Prevalence and factors associated with postpartum depression in women from single-child families. Int J Gynaecol Obstet, 141(2):194-199. doi: 10.1002/ijgo.12461. 